\title{
ESTUDIO DE LA ABUNDANCIA DE MICROPLÁSTICOS EN DOCE PLAYAS DE LA ISLA DE TENERIFE (ISLAS CANARIAS)
}

\author{
Cristina Villanova Solano*, Francisca Romero Peral, \\ Sonia Fernández Martín, Miguel Muñoz Molina \\ \& Sara Álvaro Berlanga
}

\begin{abstract}
RESUMEN
En la última década, la presencia de microplásticos en los entornos marinos se ha convertido en una importante preocupación ambiental y foco de interés de muchas investigaciones. Estos microplásticos se forman debido a la fragmentación de plásticos de mayor tamaño mediante procesos de fotodegradación y erosión. El presente estudio trata de analizar la acumulación de microplásticos en doce playas de la isla de Tenerife (Islas Canarias), analizando la capa superficial de arena a lo largo de distintos transectos establecidos en función de la amplitud de marea. Los resultados obtenidos muestran que la mayor abundancia de microplásticos fue detectada en la playa de Los Gigantes. Por el contrario, La Tejita fue la única playa que no presentó ninguna de estas partículas. Además, los microplásticos encontrados fueron principalmente del tamaño de $1 \mathrm{~mm}$, acumulándose la mayor parte de estos en la línea de bajamar. El peso registrado en ningún caso superó los $0.408 \mathrm{~g}$, siendo 0 en muchas de las playas. Estos hallazgos sugieren que la determinación cuantitativa de microplásticos que llegan a nuestras playas es muy compleja debido a la diversidad de factores que influyen, si bien podemos decir que en las playas de Tenerife existe un bajo porcentaje de microplásticos. Palabras Clave: Tenerife, playa, arena, microplásticos, marea.
\end{abstract}

\section{STUDY OF THE MICROPLASTIC ABUNDANCES IN TWELVE BEACHES OF THE TENERIFE ISLAND (CANARY ISLANDS)}

\section{Abstract}

During the last decade microplastics pollution in marine environments has become an important environmental concern and the focus of much research. In the ocean microplastic debris comes from the breakup of bigger plastic debris. The present study tries to analyze the accumulation of microplastics in twelve beaches of Tenerife (Canary Islands) by analyzing the sand surface along different transects established according to the tidal range. Our results showed that the Los Gigantes beach had the greatest abundance of microplastics, whereas La Tejita was the only beach that did not present microplastics. The microplastics that where found were primarily $1 \mathrm{~mm}$ fractions that accumulated on the lowest tide line. The microplastic weight registered never exceeded $0.408 \mathrm{~g}$; on most of the beaches, the weight was 0 . These discoveries suggest that the quantitative prediction of microplastic arrival to our beaches is very complex due to the diversity of influencing factors. According to our results, we can say that Tenerife beaches have low amounts of microplastics pollutions.

Keywords: Tenerife, beach, sand, microplastics, tide. 


\section{INTRODUCCIÓN}

Los plásticos han tomado un papel esencial en la vida diaria de las personas debido a su bajo coste de producción y la versatilidad de usos que presentan. Se calcula que los plásticos suponen entre el 60 y el 80\% de la basura marina (Derraik 2002), distribuyéndose a lo largo de todos los océanos, mares y zonas costeras del mundo, afectando negativamente al buen estado ambiental y generando graves problemas ambientales debido a su durabilidad y flotabilidad, además de su capacidad para absorber otras sustancias tóxicas. La fotodegradación y la erosión de estos plásticos en fragmentos más pequeños, microplásticos, facilitan su dispersión con las corrientes oceánicas (Escolástico y Pérez 2014), suponiendo un grave riesgo para los organismos marinos, acumulándose y transfiriéndose a lo largo de las cadenas tróficas.

El término microplásticos hace referencia a aquellos que tienen un tamaño inferior a los $5 \mathrm{~mm}$ (Andrady 2011). Estos se convierten en uno de los contaminantes más comunes y persistentes del mar y las playas de todo el mundo (Derraik 2002; Moore 2008; Ryan et al. 2009; Cózar et al. 2014; Eriksen et al. 2014). Los lugares de acumulación son numerosos y están vinculados en muchas ocasiones a las zonas más antropizadas y de menor energía hidrodinámica (Rojo-Nieto y Montoto 2017).

Las Islas Canarias es una de las áreas afectadas por este tipo de contaminación, ya que se encuentran situadas en el extremo oriental de una de una de las cinco regiones donde las corrientes superficiales impulsadas por el viento causan la convergencia de los desechos marinos (Lumpkin et al. 2012). Según Baztan et al. 2014, esta contaminación alcanza valores de hasta $100 \mathrm{~g} / \mathrm{L}$ de arena debido principalmente a la Corriente de Canarias, que trae esta contaminación desde el océano Atlántico abierto y la deposita en sus costas, principalmente en las playas con orientación norte y noreste (Baztan et al. 2014; Herrera et al. 2017).

Debido al fuerte impacto negativo que están generando los microplásticos y el gran desconocimiento de estos en la isla de Tenerife es de esencial importancia conocer su abundancia, ya que no se han realizado estudios previos como sí se hicieron para otras islas pertenecientes al archipiélago canario como Lanzarote, Fuerteventura, La Graciosa y Gran Canaria (Baztan et al. 2014; Herrera et al. 2017). Por ello en este estudio se pretende cuantificar el número y peso de microplásticos que llegan a las playas de la isla de Tenerife (Islas Canarias) y discutir las posibles causas que originan estas diferencias.

* Máster de Biología Marina: Biodiversidad y Conservación. Universidad de La Laguna, San Cristóbal de La Laguna, Tenerife, islas Canarias, España. Autor para la correspondencia: cris13_30@hotmail.com. 


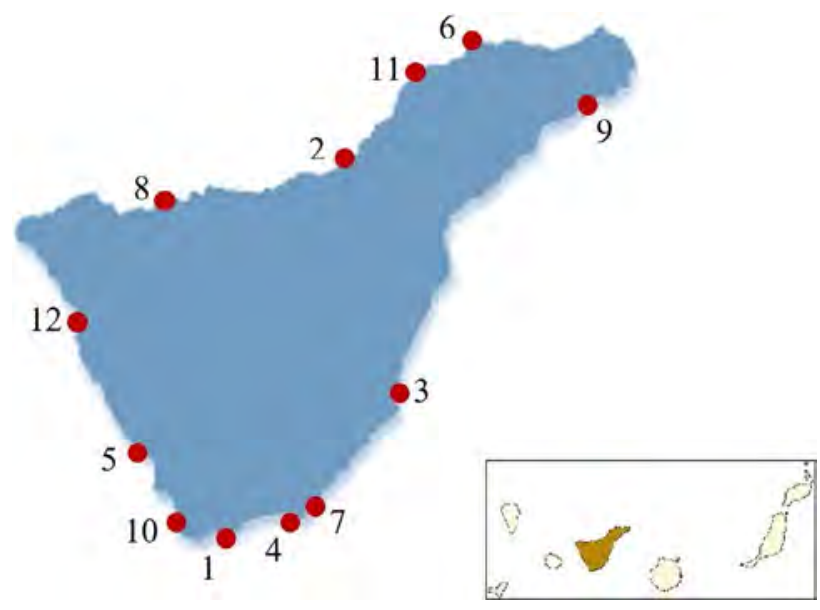

Figura 1. Áreas de estudio de la isla de Tenerife. 1. Las Galletas, 2. Playa Jardín, 3. Porís de Abona, 4. La Tejita, 5. Caleta de Adeje, 6. Punta del Hidalgo, 7. El Médano, 8. Playa San Marcos,

9. Las Gaviotas, 10. El Palm-Mar, 11. Finca El Apio y 12. Playa de Los Gigantes.

\section{MATERIAL Y MÉTODOS}

\section{1. ÁREA DE ESTUDIO}

El presente estudio se realizó durante el mes de febrero de 2018 en doce playas de arena de la isla de Tenerife (Islas Canarias) (figura 1). Esta distribución fue establecida para discernir la posible influencia de las corrientes sobre las distintas orientaciones de la isla.

\subsection{Metodología}

En cuanto a microplásticos en sedimento, existe una carencia de metodologías estandarizadas o de referencia, lo que es un punto débil. España está trabajando para subsanar este problema, en colaboración con otros países, en el marco del grupo técnico para basuras marinas, dentro de estas los microplásticos en playas creado por la Comisión Europea (TSG-ML). (CONAMA 2016). Se ha aplicado el protocolo de muestreo TSG-ML ligeramente modificado.

\subsection{TOMa DE muestras}

Se recolectaron muestras de arena en cada playa estudiada a lo largo de tres transectos de 10 metros de longitud y 1.60 metros de anchura paralelos a la línea de costa. Fueron realizados durante la amplitud de marea (diferencia vertical entre 
la marea alta y la marea baja) estableciendo el punto bajamar como referencia, por lo que los tres transectos quedaron establecidos como: línea de bajamar (transecto 1), transecto intermedio (transecto 2) y línea de pleamar (transecto 3).

Dentro de cada transecto se realizaron tres cuadrantes de $20 \times 20 \mathrm{~cm}$, distribuidos de forma aleatoria, de los cuales se recolectó la capa superficial de arena para recoger los restos depositados por la última marea.

\subsection{TOMA DE DATOS}

Las muestras de arena recolectadas fueron tratadas en el laboratorio mediante decantación, tomando $450 \mathrm{~g}$ de cada muestra en $1 \mathrm{~L}$ de agua durante 48 horas. Posteriormente se filtró el sobrenadante mediante tres tamices (de $4 \mathrm{~mm}, 1 \mathrm{~mm}$ y $0.5 \mathrm{~mm}$ de luz de malla) para separar los plásticos recogidos en función de su tamaño.

\subsection{Análisis estadístico}

Todos los análisis estadísticos realizados se llevaron a cabo con el programa estadístico R-project (R Core Team 2016). El análisis utilizado fue el análisis de la varianza (ANOVA) de las variables 'Número de microplásticos' y 'Peso de microplásticos'. Por lo tanto, los modelos incorporaron los siguientes factores: 'Playa', 'Transecto' y 'Tamiz'. El nivel de significación elegido fue de 0.05 y como test $a$ posteriori se utilizó SNK.

\section{RESULTADOS}

\subsection{ANÁLISIS DE LA ABUNDANCIA DE MICROPLÁSTICOS EN FUNCIÓN DEL NÚMERO}

Según el análisis ANOVA para la variable dependiente «Número de microplásticos», la triple interacción de transecto, playa y tamiz fue significativa (tabla 1).

TABLA 1. RESULTADOS DEL ANÁLISIS DE VARIANZA (ANOVA) CON 3 FACTORES (PLAYA, TRANSECTO Y TAMIZ), PARA EL NÚMERO DE MICROPLÁSTICOS

\begin{tabular}{lccccc}
$\begin{array}{l}\text { Fuente DE VARIACión: } \\
\text { Número DE Microplásticos } \\
\text { Alfa 0.05 }\end{array}$ & df & Sum Sq & Mean Sq & F value & $\operatorname{Pr}(>\mathrm{F})$ \\
\hline Transecto & 2 & 34.49 & 17.24 & 3.50 & $0.031^{*}$ \\
\hline Playa & 11 & 448.26 & 40.75 & 8.28 & $4.468 \mathrm{e}-12^{* * *}$ \\
\hline Tamiz & 2 & 43.60 & 21.79 & 4.43 & $0.013^{*}$ \\
\hline Transecto $\times$ playa & 22 & 526.62 & 23.93 & 4.86 & $2.555 \mathrm{e}-10$ *** \\
\hline Transecto $\times$ tamiz & 4 & 27.42 & 6.85 & 1.39 & 0.238
\end{tabular}



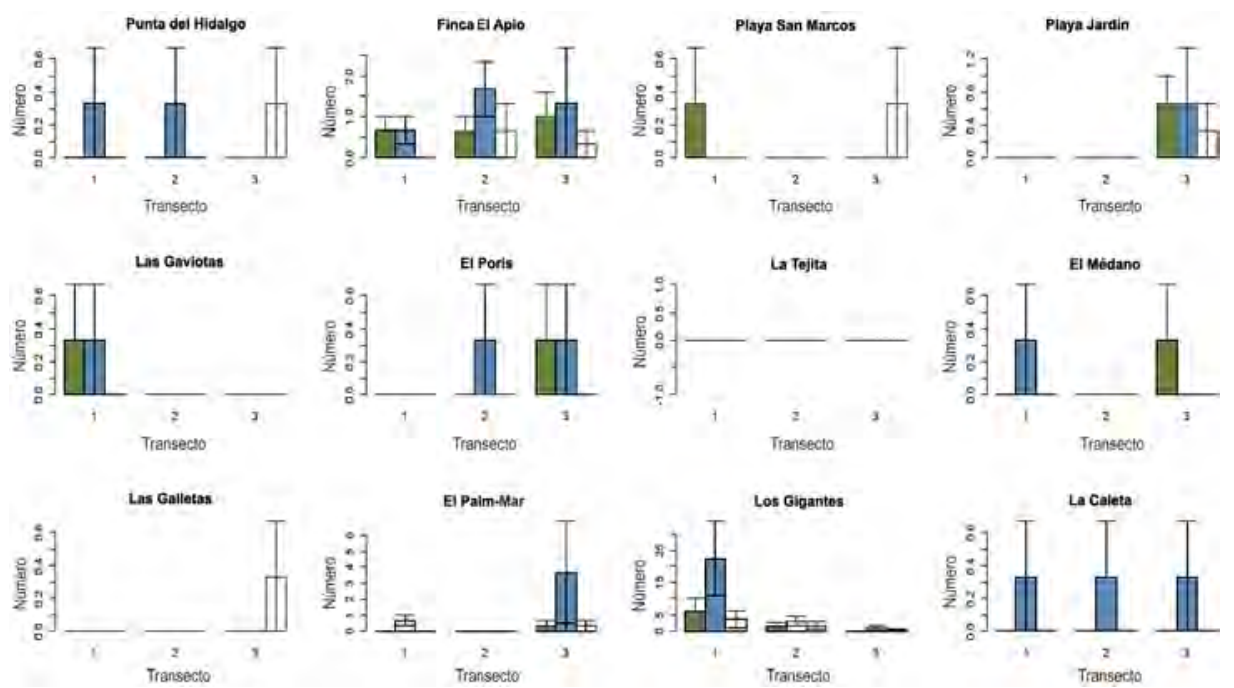

Figura 2. Número de microplásticos por playa en función del transecto y el tamiz.

Barra de errores = Errores estándar. La escala del eje Y se encuentra adaptada al número de microplásticos encontrados en cada playa.

\begin{tabular}{lccccc}
\hline Playa $\times$ tamiz & 22 & 235.73 & 10.71 & 2.18 & $0.002{ }^{* *}$ \\
\hline Transecto $\times$ playa $\times$ tamiz & 44 & 351.02 & 7.98 & 1.62 & $0.013^{*}$ \\
\hline Residual & 216 & 1063.33 & 4.92 & & \\
\hline
\end{tabular}

df: grados de libertad; Sum sq: suma de cuadrados; Mean sq: cuadrados medio; F value: estadístico; $\operatorname{Pr}>(\mathrm{F}): \mathrm{p}$-valor.

Los niveles de significación fueron: $\left.{ }^{*} \mathrm{p}<0.05,{ }^{* *} \mathrm{p}<0.01 \mathrm{y}{ }^{* * *} \mathrm{p}<0.001\right)$

El test a posteriori (Anexo) mostró que el número de microplásticos para el transecto 1 fue significativamente mayor para la playa de Los Gigantes con respecto al resto de playas, mientras que los transectos 2 y 3 no mostraron diferencias significativas entre las distintas playas. Además, solo la playa de Los Gigantes mostró diferencias significativas entre los distintos transectos, existiendo esas diferencias en el transecto 3 con respecto al 1 y 2 .

En vista de los resultados (figura 2), el mayor número de microplásticos encontrados se dio en playa de Los Gigantes, mientras que en playa de La Tejita no se obtuvo presencia de estos.

En siete de las doce playas examinadas no se detectó presencia de microplásticos en el transecto 2 . Sin embargo, en aquellas en las que sí aparecían en este transecto el número de ellos se dio en un rango similar o superior a los otros dos.

El tamaño predominante de microplásticos fue de $1 \mathrm{~mm}$. No obstante, cabe destacar que la mayoría de microplásticos de tamaño $4 \mathrm{~mm}$ solo se encontraron en el transecto 3, a excepción de playa de Los Gigantes en la que estaban presentes en todos los transectos, y Finca El Apio donde también aparecían en el transecto 2. En la playa de La Caleta solo se encontraron los pertenecientes a la fracción de $1 \mathrm{~mm}$. 
Según el análisis ANOVA para la variable dependiente «Peso de microplásticos», la interacción de transecto y playa fue significativa (tabla 2).

\begin{tabular}{|c|c|c|c|c|c|}
\hline \multicolumn{6}{|c|}{$\begin{array}{l}\text { TABLA 2. RESULTADOS DEL ANÁLISIS DE VARIANZA (ANOVA) CON } 3 \text { FACTORES } \\
\text { (PLAYA, TRANSECTO Y TAMIZ), PARA EL PESO DE MICROPLÁSTICOS }\end{array}$} \\
\hline $\begin{array}{l}\text { FuENTE DE VARIACIÓN: } \\
\text { PESO DE MICROPLÁSTICOS } \\
\text { ALFA } 0.05\end{array}$ & df & Sum Sq & Mean Sq & F value & $\operatorname{Pr}(>\mathrm{F})$ \\
\hline Transecto & 2 & 20.41 & 10.20 & 0.83 & 0.435 \\
\hline Playa & 11 & 780.77 & 70.98 & 5.81 & $3.158 \mathrm{e}-08^{* * *}$ \\
\hline Tamiz & 2 & 119.45 & 59.72 & 4.89 & $0.008^{* *}$ \\
\hline Transecto $\times$ playa & 22 & 452.03 & 20.54 & 1.68 & $0.032 *$ \\
\hline Transecto $\times$ tamiz & 4 & 43.33 & 10.83 & 0.88 & 0.473 \\
\hline Playa $\times$ tamiz & 22 & 400.99 & 18.22 & 1.49 & 0.078 \\
\hline Transecto $\times$ playa $\times$ tamiz & 44 & 293.34 & 6.66 & 0.54 & 0.991 \\
\hline Residual & 216 & 2638.67 & 12.21 & & \\
\hline
\end{tabular}

df: grados de libertad; Sum sq: suma de cuadrados; Mean sq: cuadrados medio; F value: estadístico; $\operatorname{Pr}>(\mathrm{F})$ : p-valor

Los niveles de significación fueron: $\left.{ }^{*} \mathrm{p}<0.05,{ }^{* *} \mathrm{p}<0.01 \mathrm{y}{ }^{* * *} \mathrm{p}<0.001\right)$.

El test a posteriori (Anexo tablas de la 3 a la 10) mostró diferencias significativas entre playas, destacando el transecto 1 y 2 de la playa de Los Gigantes. También se encontraron diferencias en el transecto 3 de playa Jardín con respecto a La Caleta, Las Gaviotas, El Médano, San Marcos y La Tejita.

En cuanto a diferencias entre los transectos realizados para cada playa, destacar el transecto 3 de playa Jardín y el transecto 1 de playa de Los Gigantes, al ser significativamente diferentes a los otros transectos realizados en la misma playa.

Conforme a los resultados obtenidos (figura 3) podemos observar que las mayores abundancias de microplásticos fueron encontradas en la playa de Los Gigantes y Finca El Apio. Por otro lado, cuatro de las playas (Las Gaviotas, San Marcos, La Tejita y El Médano) presentaron un peso de $0 \mathrm{~g}$.

En las playas cuyo peso fue distinto de $0 \mathrm{~g}$, hubo cinco en las que el peso solo se distribuyó a lo largo de uno de los transectos, dándose en cuatro de ellas en el transecto 3, mientras que para la playa de La Caleta solo se dieron únicamente en el transecto 2 .

Para las playas de Los Gigantes, Finca El Apio y El Palm-Mar sí encontramos el peso de microplásticos distribuido entre varios de los transectos, donde para Los Gigantes y Finca El Apio la mayor parte del peso se concentró en el transecto 2, mientras que para la playa El Palm-Mar el transecto predominante fue el 3. 

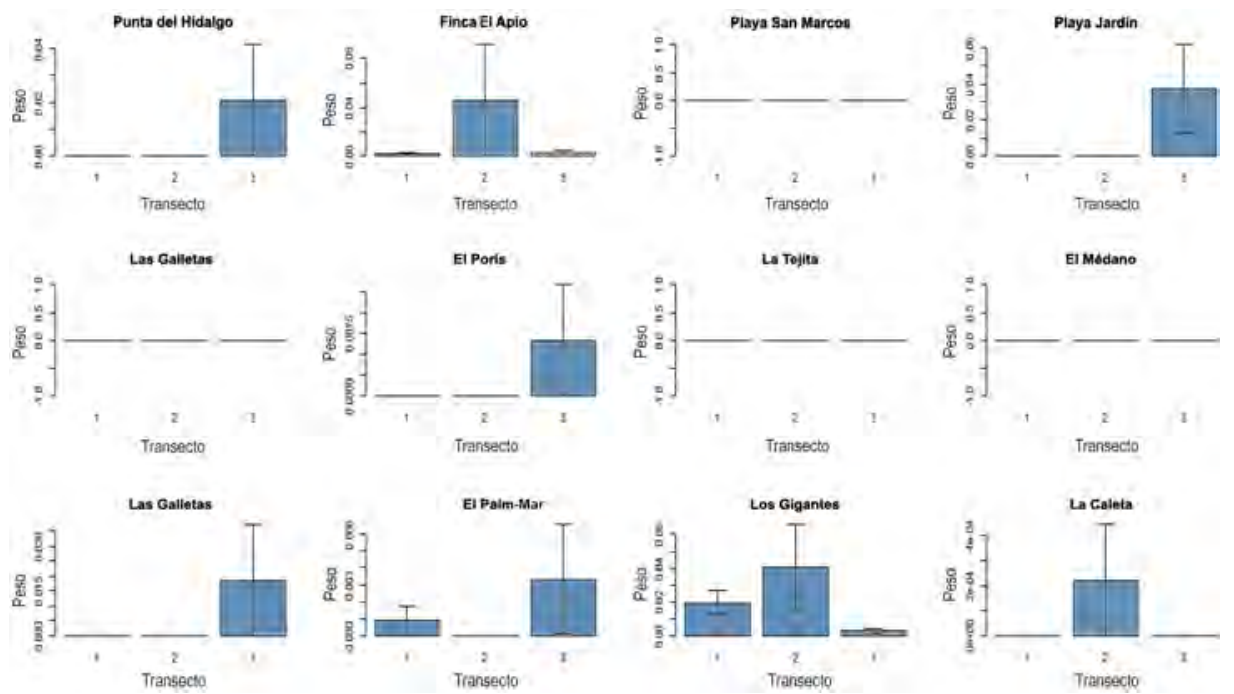

Figura 3. Peso de microplásticos por playa en función del transecto. Barra de errores = Errores estándar. La escala del eje Y se encuentra adaptada al peso de microplásticos encontrados en cada playa.

\section{DISCUSIÓN}

El presente trabajo conforma un estudio pionero sobre la cuantificación de microplásticos en las diferentes playas de la isla de Tenerife (Islas Canarias), el cual ha revelado que existe una relación entre la abundancia de microplásticos y la interacción entre los distintos factores evaluados. El número de microplásticos está condicionado por la interacción entre la playa, el transecto y el tamiz; mientras que el peso viene determinado por la interacción entre playa y transecto, sin que el tamaño de los microplásticos resulte relevante.

Conforme a los resultados obtenidos se ha podido observar que el número de microplásticos encontrados fue muy superior en la playa de Los Gigantes, siendo a su vez la que mayor peso de microplásticos reflejó. Por otro lado, La Tejita fue la única playa que no presentó microplásticos. Esta variabilidad puede ser debida a las características propias de cada playa como su morfología, viento, oleaje, mareas y corrientes. Sin embargo, se desconoce en qué medida están influyendo estas propiedades en el transporte y en los patrones espaciales de distribución de los fragmentos de plásticos (Rojo-Nieto y Montoto 2017). En relación con ello, diversos autores han demostrado que la Corriente de Canarias es casi insignificante en invierno, ya que esta sigue un patrón estacional fluyendo próxima a la costa africana durante la primavera, a través de todo el archipiélago canario durante el verano donde se intensifica y se mueve hacia las islas occidentales durante el otoño (Machín et al. 2006; Mason et al. 2011). Debido a ello, en nuestro estudio la variabilidad observada puede ser debida 
no a la Corriente de Canarias, sino a la presencia de temporales durante la época de invierno, ya que el muestreo se realizó durante el mes de febrero, quedando las playas de Tenerife condicionadas por estos temporales, donde aquellas que se sitúan en el este y oeste sufren los temporales provenientes del sudoeste mientras que las situadas en la región norte estarán sujetas a los que provienen del norte y noroeste (Plan Insular de Ordenación de Tenerife 2011). Sería necesario realizar un muestreo en otra época del año permitiéndonos observar posibles patrones estacionales ocasionados por las distintas variables ambientales como los temporales o los vientos alisios a lo largo del ańo, corroborando así los datos obtenidos.

La cantidad de plásticos flotando en los océanos será determinante a la hora de evaluar su abundancia en nuestras costas. Estimaciones sobre el total de residuos plásticos flotantes demostraron que el $92.4 \%$ de ellos pertenecen a la categoría de los microplásticos, muchos de ellos provienen de la ruptura de piezas de plástico mayores durante su deriva, por lo que es esperable la mayor abundancia de estas piezas de menor tamaño (Eriksen et al. 2014; Escolástico y Pérez 2014). Nuestros resultados obtenidos mostraron que el tamaño más representativo fue de 1 a $4 \mathrm{~mm}$, seguido de 0.5 a $1 \mathrm{~mm}$ y, por último, mayores a $4 \mathrm{~mm}$; datos ya verificados anteriormente por Eriksen et al. 2014, donde se encontró que los microplásticos pertenecientes a la fracción entre los 1 y $4.75 \mathrm{~mm}$ resultaron un $40 \%$ más abundantes que los menores de $1 \mathrm{~mm}$.

Respecto al peso, en ninguno de los casos se superaron los $0.408 \mathrm{~g}$. Incluso, algunos microplásticos no permitieron obtener resultados sobre el peso al darse por debajo del límite de cuantificación de la balanza utilizada $(0.001 \mathrm{~g})$.

En relación con la distribución de los microplásticos en las playas, los resultados obtenidos han demostrado que la mayoría de los microplásticos superiores a 1 $\mathrm{mm}$ se encontraron en el nivel de pleamar (transecto 3). Esto puede ser debido a que al tratarse de fragmentos de mayor tamaño y por consiguiente los que mayor peso alcanzan, requieren de un mayor esfuerzo del agua para arrastrarlos, donde una vez alcanzado el límite de pleamar es más difícil que vuelvan a ser removilizados por el reflujo. A su vez, esto también explicaría por qué los de menor tamaño, y con ello los de menor peso, sí pueden volver a ser transportados por el reflujo hasta el transecto 1 , siendo los microplásticos de esta fracción los más abundantes.

Es difícil establecer una imagen cuantitativa de la presencia de microplásticos en las playas, ya que los factores que influyen en su evaluación y cuantificación son muchos, principalmente el uso de distintas técnicas de muestreo y recuento, que no permiten comparar datos fácilmente (Rojo-Nieto y Montoto 2017).

Una vez concluido el estudio, los hallazgos han sugerido que las playas de la isla de Tenerife analizadas durante el mes de febrero han presentado muy bajo porcentaje en cuanto a contaminación por microplásticos. 


\section{AGRADECIMIENTOS}

Queremos agradecer al Dr. José Carlos Hernández la ayuda, tutorización prestada a la hora de realizar este trabajo y los materiales aportados, y cómo no, dar las gracias al Departamento de Biología Animal, Edafología y Geología de la ULL, en especial al Dr. Julio de la Nuez por los tamices prestados.

\section{CONTRIBUCIÓN DE LOS AUTORES}

Conceptualización: CV.

Metodología y trabajo de campo: CV, FR, SF, MM, SA.

Análisis de datos: CV, FR, SF, MM, SA.

Preparación del escrito original: CV, FR, SF, MM, SA.

Corrección y edición del escrito definitivo: CV, FR, SF, MM, SA. 


\section{REFERENCIAS}

Andrady, A.L. 2011. Microplastics in the marine environment. Mar. Pollut. Bull. 62(8): 1596-1605.

Baztan, J., Carrasco, A., Chouinard, O., Cleaud, M., Gabaldon, J.E., Huck, T., Jaffrès, L., Jorgensen, B., Miguelez, A., Paillard, C. y Vanderlinden, J.P. 2014. Protected areas in the Atlantic facing the hazards of micro-plastic pollution: first diagnosis of three islands in the Canary Current. Mar. Pollut. Bull. 80(1-2): 302-311.

Cabildo de Tenerife. 2011. Plan Insular de Ordenación de Tenerife 2011.Información ambiental. Caracterización del Litoral Insular. Puertos. Estudios de base. [Internet]. Avaiable from: http://www.tenerife.es/planes/PIOT/adjuntos/ADef_E-Base_Puertos_Feb2011_8.pdf.

Cózar, A., Echevarría, F., González-Gordillo, J.I., Irigoien, X., Úbeda, B., Hernández-León, S., Palma, A.T., Navarro, S., García-de Lomas, J., Ruiz, A., Fernández-de-Puelles, M.L. y Duarte, C.M. 2014. Plastic debris in the open ocean. PNAS. 111(28): 10239-10244.

Derraik, J.G.B. 2002. Depollution of the marine environment by plastic debris: a review. Mar. Pollut. Bull. 44(9): 842-852.

Escolástico León, C. y Pérez Esteban, J. 2014. Novedades científicas en 2014 en Ciencias Ambientales.100cias@UNED.7:60-65.

Eriksen, M., Lebreton, L.C.M., Carson, H.S., Thiel, M., Moore, C.J., Borerro, J.C., GalgaNI, F., Ryan, P.G. y Reisser, J. 2014. Plastic pollution in the world's oceans: more than 5 trillion plastic pieces weighing over 250,000 tons afloat at sea. PLoS ONE. 9(12): e111913.

Fundación CONAMA. 2016. Basuras marinas. Congreso Nacional del Medio Ambiente (CONAMA). Available from http://www.conama.org/conama/download/files/conama2016/ GTs\%202016/16_final.pdf.

Herrera, A., Asensio, M., Martínez, I., Santana, A., Packard, T. y Gómez, M. 2017. Microplastic and tar pollution on three Canary Islands beaches: an annual study. Mar. Pollut. Bull. [Internet]. Disponible en https://www.sciencedirect.com/science/article/pii/ S0025326X1730838X.

Lumpkin, R., Maximenko, N. y Pazos, M. 2012. Evaluating where and why drifters die. J. Atmos. Oceanic Technol. 29(2): 300-308.

Machin, F., Hernández-Guerra, A. y Pelegrí, J.L. 2006. Mass fluxes in the Canary Basin. Prog. Oceanogr. 70(2-4):416-447.

Mason, E., Colas, F., Molemaker, J., Shchepetkin, A.F., Troupin, C., McWilliams, J.C. y SANGrÀ, P. 2011. Seasonal variability of the Canary Current: a numerical study. J. Geophys. Res. 116(C6).

Moore, C.J. 2008. Synthetic polymers in the marine environment: a rapidly increasing, long-term threat. Environ. Res. 108(2): 131-139.

R Core Team. 2016. R: A language and environment for statistical computing [Internet]. T Foundation for Statistical Computing, Vienna, Austria. Available from http://www.Rproject.org/.

Rojo-Nieto, E. у Montoto, T. 2017. Basuras marinas, plásticos y microplásticos: orígenes, impactos y consecuencias de una amenaza global. Ecologistas en Acción. Available from https://www. ecologistasenaccion.org/IMG/pdf/informe-basuras-marinas.pdf.

Ryan, P.G., Moore, C.J., van Franeker, J.A. y Moloney, C.L. 2009. Monitoring the abundance of plastic debris in the marine environment. Phil. Trans. R. Soc. B. 364(1526): 1999-2012. 


\section{ANEXO}

\begin{tabular}{|c|c|c|c|c|c|c|c|c|c|c|c|c|}
\hline TABLA 3. & ESULT & $\begin{array}{l}\text { ADOS } \\
\text { PARES } \\
\text { PARA }\end{array}$ & $\begin{array}{l}\text { NTR } \\
\text { A V }\end{array}$ & $\begin{array}{l}\text { ST } A \\
\text { PLAY } \\
\text { IABI }\end{array}$ & $\begin{array}{l}\text { OSTER } \\
\text { S DEN } \\
\text { NÚMP }\end{array}$ & $\begin{array}{l}\text { IORI } \\
\text { TRO D } \\
\text { ERO D }\end{array}$ & $\begin{array}{l}\text { NK.TE } \\
\text { E CAD } \\
\text { E MICR }\end{array}$ & $\begin{array}{l}\text { ST) DE } \\
\text { 4 TRAI } \\
\text { OPLÁS }\end{array}$ & $\begin{array}{l}\text { LA CO } \\
\text { NSECT } \\
\text { TICOS }\end{array}$ & $\begin{array}{l}\text { MPAF } \\
\mathrm{O}\end{array}$ & CIĆ & \\
\hline & & & & & Nús & AERO & & & & & & \\
\hline & & SNI & TES & NTRE & LAYAS D & ENTRO & DE CAD & TRANSE & СTO & & & \\
\hline Transecto 1 & $\underset{\Xi}{\stackrel{\Xi}{\Xi}}$ & 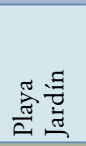 & 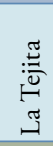 & $\underset{\widetilde{\pi}}{\frac{\pi}{\tilde{U}}}$ & 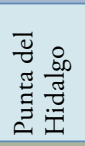 & 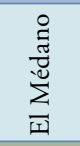 & 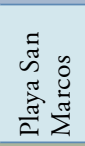 & 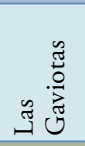 & 四 & 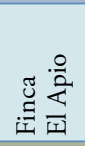 & ڤ & 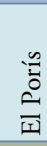 \\
\hline Las Galletas & - & ns & ns & ns & ns & ns & ns & ns & ns & ns & $* * *$ & ns \\
\hline Playa Jardín & ns & - & ns & ns & $\mathrm{ns}$ & ns & ns & $\mathrm{ns}$ & ns & $\mathrm{ns}$ & $* * *$ & ns \\
\hline La Tejita & ns & ns & - & ns & ns & ns & ns & ns & ns & ns & $* * *$ & ns \\
\hline La Caleta & ns & ns & ns & - & ns & ns & ns & ns & ns & ns & $* * *$ & ns \\
\hline $\begin{array}{l}\text { Punta del } \\
\text { Hidalgo }\end{array}$ & ns & ns & ns & ns & - & ns & ns & ns & ns & ns & $* * *$ & ns \\
\hline El Médano & ns & ns & ns & ns & ns & - & ns & ns & ns & ns & $* * *$ & ns \\
\hline $\begin{array}{l}\text { Playa San } \\
\text { Marcos }\end{array}$ & ns & ns & ns & ns & ns & ns & - & ns & ns & ns & $* * *$ & ns \\
\hline Las Gaviotas & ns & ns & ns & ns & ns & ns & ns & - & ns & ns & $* * *$ & ns \\
\hline El Palm-Mar & ns & ns & $\mathrm{ns}$ & ns & ns & ns & ns & ns & - & ns & $* * *$ & ns \\
\hline Finca El Apio & ns & $\mathrm{ns}$ & $\mathrm{ns}$ & ns & $\mathrm{ns}$ & ns & ns & ns & $\mathrm{ns}$ & - & $* * *$ & ns \\
\hline Los Gigantes & $* * *$ & $* * *$ & $* * *$ & $* * *$ & $* * *$ & $* * *$ & $* * *$ & $* * *$ & $* * *$ & $* * *$ & - & *** \\
\hline El Porís & ns & ns & ns & ns & ns & $\mathrm{ns}$ & $\mathrm{ns}$ & ns & $\mathrm{ns}$ & ns & $* * *$ & - \\
\hline Transecto 2 & 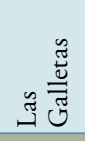 & 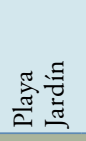 & : & $\underset{\widetilde{\pi}}{\frac{\pi}{\tilde{U}}}$ & 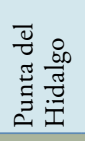 & 푀 & 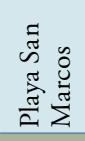 & 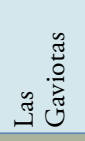 & 牙 & 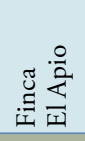 & 总 & 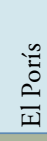 \\
\hline Las Galletas & - & ns & ns & ns & ns & ns & $\mathrm{ns}$ & ns & ns & ns & ns & ns \\
\hline Playa Jardín & $\mathrm{ns}$ & - & ns & ns & ns & ns & ns & ns & $\mathrm{ns}$ & ns & ns & ns \\
\hline La Tejita & ns & ns & - & ns & ns & ns & ns & ns & ns & ns & ns & ns \\
\hline La Caleta & ns & ns & ns & - & ns & ns & ns & ns & ns & ns & ns & ns \\
\hline $\begin{array}{l}\text { Punta del } \\
\text { Hidalgo }\end{array}$ & ns & ns & ns & ns & - & ns & ns & ns & ns & ns & ns & ns \\
\hline El Médano & ns & $\mathrm{ns}$ & ns & ns & ns & - & ns & ns & ns & ns & ns & ns \\
\hline $\begin{array}{l}\text { Playa San } \\
\text { Marcos }\end{array}$ & ns & ns & ns & ns & ns & ns & - & ns & ns & ns & ns & ns \\
\hline Las Gaviotas & $\mathrm{ns}$ & ns & $\mathrm{ns}$ & ns & ns & ns & ns & - & ns & ns & ns & ns \\
\hline El Palm-Mar & ns & ns & $\mathrm{ns}$ & ns & ns & ns & ns & ns & - & ns & ns & ns \\
\hline Finca El Apio & ns & ns & $\mathrm{ns}$ & ns & ns & ns & ns & ns & ns & - & ns & ns \\
\hline Los Gigantes & ns & ns & $\mathrm{ns}$ & ns & ns & ns & ns & $\mathrm{ns}$ & ns & ns & - & ns \\
\hline El Porís & ns & ns & ns & ns & ns & ns & ns & ns & ns & ns & ns & - \\
\hline
\end{tabular}




\begin{tabular}{|c|c|c|c|c|c|c|c|c|c|c|c|c|}
\hline Transecto 3 & 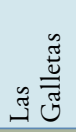 & 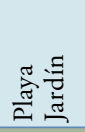 & $\frac{\sqrt[\pi]{\pi}}{:=0}$ & $\underset{\stackrel{\pi}{త}}{\frac{\pi}{\tilde{J}}}$ & 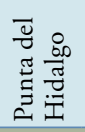 & 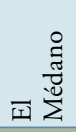 & 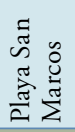 & 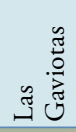 & 四 & 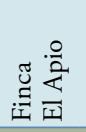 & 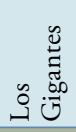 & $\frac{\ddots}{0}$ \\
\hline Las Galletas & - & ns & $\mathrm{ns}$ & $\mathrm{ns}$ & ns & ns & ns & ns & ns & ns & ns & ns \\
\hline Playa Jardín & ns & - & ns & ns & ns & ns & ns & ns & ns & ns & ns & ns \\
\hline La Tejita & ns & ns & - & ns & ns & ns & ns & ns & ns & ns & ns & ns \\
\hline La Caleta & ns & ns & ns & - & ns & ns & ns & ns & ns & ns & ns & ns \\
\hline $\begin{array}{l}\text { Punta del } \\
\text { Hidalgo }\end{array}$ & ns & ns & ns & ns & - & ns & ns & ns & ns & ns & ns & ns \\
\hline El Médano & ns & ns & ns & ns & ns & - & ns & ns & ns & ns & ns & $\mathrm{ns}$ \\
\hline $\begin{array}{l}\text { Playa San } \\
\text { Marcos }\end{array}$ & ns & ns & ns & ns & ns & ns & - & ns & ns & ns & ns & ns \\
\hline Las Gaviotas & ns & ns & ns & ns & ns & ns & ns & - & ns & ns & ns & ns \\
\hline El Palm-Mar & ns & ns & ns & $\mathrm{ns}$ & ns & ns & ns & ns & - & ns & ns & ns \\
\hline Finca El Apio & ns & ns & ns & ns & ns & ns & ns & ns & ns & - & ns & ns \\
\hline Los Gigantes & ns & ns & ns & ns & ns & ns & ns & ns & ns & ns & - & ns \\
\hline El Porís & ns & ns & ns & ns & ns & ns & ns & ns & ns & ns & ns & - \\
\hline
\end{tabular}

Los niveles de significación fueron: ns $\left.\mathrm{p}>0.05,{ }^{*} \mathrm{p}<0.05,{ }^{* *} \mathrm{p}<0.01 \mathrm{y}{ }^{* * *} \mathrm{p}<0.001\right)$

\begin{tabular}{|c|c|c|c|c|c|c|c|}
\hline TABLA & $\begin{array}{l}\text { TA } \\
\text { RE }\end{array}$ & $\begin{array}{l}\text { DEL } \\
\text { RE } \\
\text { RIA }\end{array}$ & $\begin{array}{l}A P C \\
\text { SEC } \\
\text { ÚM }\end{array}$ & $\begin{array}{l}\text { ERIORI (SN } \\
\text { S DENTRO } \\
\text { O DE MICRO }\end{array}$ & $\begin{array}{l}\mathrm{DE} \\
\mathrm{AAP} \\
\mathrm{ICC}\end{array}$ & $\begin{array}{l}\mathrm{M} \\
\mathrm{AP}\end{array}$ & ÓN \\
\hline & & & & MERO & & & \\
\hline & & EST: & TRAI & CTOS DENTRO & PLA & & \\
\hline Las Galletas & 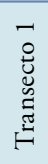 & 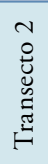 & 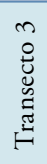 & Playa Jardín & 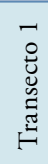 & 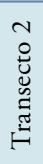 & 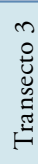 \\
\hline Transecto 1 & - & ns & ns & Transecto 1 & - & ns & ns \\
\hline Transecto 2 & ns & - & ns & Transecto 2 & ns & - & ns \\
\hline Transecto 3 & ns & ns & - & Transecto 3 & ns & ns & \\
\hline La Tejita & 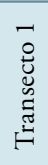 & 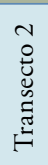 & 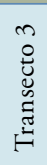 & La Caleta & 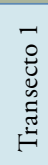 & 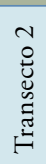 & 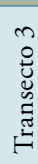 \\
\hline Transecto 1 & - & ns & ns & Transecto 1 & - & $\mathrm{ns}$ & ns \\
\hline Transecto 2 & ns & - & ns & Transecto 2 & ns & - & ns \\
\hline Transecto 3 & ns & ns & - & Transecto 3 & ns & ns & - \\
\hline
\end{tabular}




\begin{tabular}{|c|c|c|c|c|c|c|c|}
\hline Punta del Hidalgo & 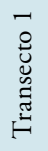 & 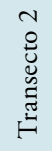 & 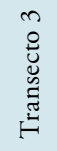 & El Médano & 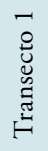 & 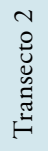 & 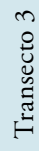 \\
\hline Transecto 1 & - & ns & ns & Transecto 1 & - & ns & ns \\
\hline Transecto 2 & ns & - & ns & Transecto 2 & ns & - & ns \\
\hline Transecto 3 & ns & ns & - & Transecto 3 & ns & ns & - \\
\hline Playa San Marcos & 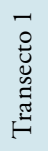 & 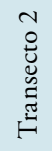 & 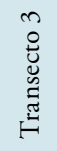 & Las Gaviotas & 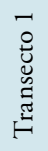 & 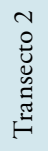 & 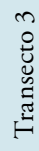 \\
\hline Transecto 1 & - & ns & ns & Transecto 1 & - & ns & ns \\
\hline Transecto 2 & ns & - & ns & Transecto 2 & ns & - & ns \\
\hline Transecto 3 & ns & ns & - & Transecto 3 & ns & ns & - \\
\hline El Palm-Mar & 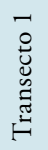 & 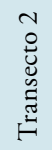 & 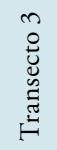 & Fina El Apio & 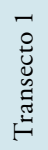 & 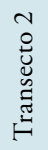 & 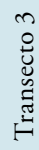 \\
\hline Transecto 1 & - & ns & ns & Transecto 1 & - & ns & ns \\
\hline Transecto 2 & ns & - & ns & Transecto 2 & ns & - & ns \\
\hline Transecto 3 & ns & ns & - & Transecto 3 & ns & ns & - \\
\hline Los Gigantes & 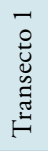 & 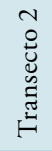 & 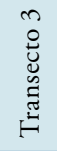 & El Porís & 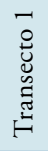 & 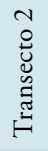 & 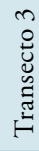 \\
\hline Transecto 1 & - & ns & $* * *$ & Transecto 1 & - & ns & $\mathrm{ns}$ \\
\hline Transecto 2 & ns & - & $* * *$ & Transecto 2 & ns & - & ns \\
\hline Transecto 3 & $* * *$ & $* * *$ & - & Transecto 3 & ns & ns & - \\
\hline
\end{tabular}

Los niveles de significación fueron: $\mathrm{ns} \mathrm{p}>0.05,{ }^{*} \mathrm{p}<0.05,{ }^{* *} \mathrm{p}<0.01 \mathrm{y}{ }^{* * *} \mathrm{p}<0.001$ ).

\begin{tabular}{|c|c|c|c|c|c|c|c|c|c|c|c|c|}
\hline TABL & $\begin{array}{r}\text { RESU } \\
\text { PO }\end{array}$ & $\begin{array}{r}\text { LTADC } \\
\text { R PARE } \\
\text { LA }\end{array}$ & LIV & E P & $\begin{array}{l}\text { POST } \\
\text { IAS DE } \\
\text { MERC }\end{array}$ & $\begin{array}{l}\text { ERIOR } \\
\text { ENTR } \\
\text { DE N }\end{array}$ & $\begin{array}{l}\text { (SNK } \\
\text { DE C } \\
\text { ICROI }\end{array}$ & $\begin{array}{l}\text { EST) } \\
\text { DA TA } \\
\text { ÁSTIC }\end{array}$ & $\begin{array}{l}\text { E LA } \\
\text { MIZ } \\
\text { OS }\end{array}$ & $\begin{array}{l}\text { OMP } \\
\text { RA }\end{array}$ & $A$ & \\
\hline & & & & & NúN & IERO & & & & & & \\
\hline & & & K.T & ENT & E PLAYAS & DENTI & $\mathrm{O}$ DE CA & DA TAM & & & & \\
\hline $\begin{array}{l}\text { Tamiz } \\
4 \mathrm{~mm}\end{array}$ & 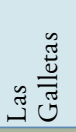 & 奈壳 & : & $\underset{\widetilde{J}}{\stackrel{\widetilde{J}}{\widetilde{J}}}$ & 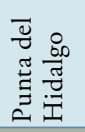 & 四 & 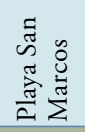 & 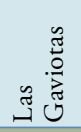 & 四 & 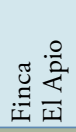 & \& & 莒 \\
\hline Las Galletas & - & ns & ns & ns & ns & ns & ns & ns & ns & ns & ns & ns \\
\hline Playa Jardín & ns & - & $\mathrm{ns}$ & ns & $\mathrm{ns}$ & ns & ns & $\mathrm{ns}$ & ns & ns & ns & $\mathrm{ns}$ \\
\hline La Tejita & ns & ns & - & ns & ns & ns & ns & ns & ns & ns & ns & ns \\
\hline La Caleta & ns & ns & ns & - & ns & ns & ns & ns & ns & ns & ns & ns \\
\hline
\end{tabular}




\begin{tabular}{|c|c|c|c|c|c|c|c|c|c|c|c|c|}
\hline $\begin{array}{l}\text { Punta del } \\
\text { Hidalgo }\end{array}$ & $\mathrm{ns}$ & $\mathrm{ns}$ & ns & $\mathrm{ns}$ & - & ns & $\mathrm{ns}$ & ns & ns & ns & ns & $\mathrm{ns}$ \\
\hline El Médano & ns & ns & ns & ns & ns & - & ns & ns & ns & ns & ns & ns \\
\hline $\begin{array}{l}\text { Playa San } \\
\text { Marcos }\end{array}$ & $\mathrm{ns}$ & $\mathrm{ns}$ & ns & $\mathrm{ns}$ & $\mathrm{ns}$ & ns & - & ns & $\mathrm{ns}$ & ns & $\mathrm{ns}$ & $\mathrm{ns}$ \\
\hline Las Gaviotas & ns & ns & ns & $\mathrm{ns}$ & ns & ns & ns & - & ns & ns & ns & ns \\
\hline El Palm-Mar & ns & ns & ns & ns & ns & ns & ns & ns & - & ns & ns & ns \\
\hline Finca El Apio & ns & ns & ns & ns & ns & ns & ns & ns & ns & - & ns & ns \\
\hline Los Gigantes & ns & ns & ns & ns & ns & ns & ns & ns & ns & ns & - & ns \\
\hline El Porís & ns & ns & $\mathrm{ns}$ & ns & ns & ns & ns & ns & ns & ns & ns & - \\
\hline $\begin{array}{l}\text { Tamiz } \\
1 \mathrm{~mm}\end{array}$ & 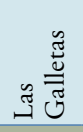 & 㞼恶 & 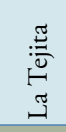 & $\underset{\widetilde{J}}{\stackrel{\widetilde{J}}{\widetilde{J}}}$ & 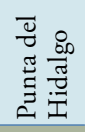 & 四 & 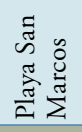 & 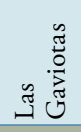 & 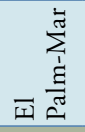 & 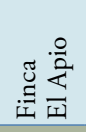 & $\stackrel{\mho}{\stackrel{\Xi}{\Xi}}$ & $\stackrel{0}{\vdots}$ \\
\hline Las Galletas & - & ns & ns & ns & ns & ns & ns & ns & ns & ns & $* * *$ & ns \\
\hline Playa Jardín & ns & - & ns & ns & ns & $\mathrm{ns}$ & ns & ns & ns & ns & $* * *$ & ns \\
\hline La Tejita & ns & ns & - & ns & ns & ns & ns & ns & ns & ns & $* * *$ & ns \\
\hline La Caleta & ns & ns & ns & - & ns & ns & ns & ns & $\mathrm{ns}$ & ns & $* * *$ & ns \\
\hline $\begin{array}{l}\text { Punta del } \\
\text { Hidalgo }\end{array}$ & ns & ns & ns & $\mathrm{ns}$ & - & $\mathrm{ns}$ & ns & $\mathrm{ns}$ & ns & $\mathrm{ns}$ & $* * *$ & ns \\
\hline El Médano & ns & ns & ns & ns & ns & - & ns & ns & ns & ns & $* * *$ & $\mathrm{~ns}$ \\
\hline $\begin{array}{l}\text { Playa San } \\
\text { Marcos }\end{array}$ & ns & ns & $\mathrm{ns}$ & $\mathrm{ns}$ & $\mathrm{ns}$ & ns & - & $\mathrm{ns}$ & ns & ns & $* * *$ & $\mathrm{~ns}$ \\
\hline Las Gaviotas & ns & ns & ns & ns & ns & ns & ns & - & ns & ns & $* * *$ & ns \\
\hline El Palm-Mar & ns & ns & $\mathrm{ns}$ & ns & ns & ns & ns & ns & - & ns & $* * *$ & ns \\
\hline Fina El Apio & ns & ns & ns & ns & ns & ns & ns & ns & ns & - & $* * *$ & ns \\
\hline Los Gigantes & $* * *$ & $* * *$ & $* * *$ & $* * *$ & $* * *$ & $* * *$ & $* * *$ & $* * *$ & $* * *$ & $* * *$ & - & $* * *$ \\
\hline El Porís & ns & ns & ns & ns & $\mathrm{ns}$ & ns & ns & ns & ns & ns & $* * *$ & - \\
\hline $\begin{array}{l}\text { Tamiz } \\
0.5 \mathrm{~mm}\end{array}$ & 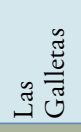 & 荵売 & : & $\underset{\widetilde{J}}{\frac{\pi}{\tilde{U}}}$ & 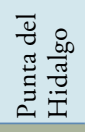 & 四总 & 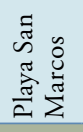 & 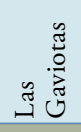 & 포 & 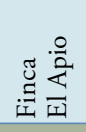 & $\stackrel{\mathscr{\Xi}}{\stackrel{\Xi}{\Xi}}$ & $\stackrel{\cong}{\Xi}$ \\
\hline Las Galletas & - & ns & ns & ns & ns & ns & ns & ns & ns & ns & ns & ns \\
\hline Playa Jardín & ns & - & ns & ns & ns & ns & ns & ns & ns & ns & ns & $\mathrm{ns}$ \\
\hline La Tejita & ns & ns & - & ns & ns & ns & ns & ns & ns & ns & ns & ns \\
\hline La Caleta & ns & ns & ns & - & ns & ns & ns & ns & ns & ns & ns & ns \\
\hline $\begin{array}{l}\text { Punta del } \\
\text { Hidalgo }\end{array}$ & ns & ns & ns & $\mathrm{ns}$ & - & $\mathrm{ns}$ & $\mathrm{ns}$ & $\mathrm{ns}$ & ns & $\mathrm{ns}$ & ns & $\mathrm{ns}$ \\
\hline El Médano & ns & ns & ns & ns & ns & - & ns & ns & ns & ns & ns & ns \\
\hline $\begin{array}{l}\text { Playa San } \\
\text { Marcos }\end{array}$ & ns & ns & ns & $\mathrm{ns}$ & ns & ns & - & ns & ns & ns & ns & ns \\
\hline Las Gaviotas & ns & ns & ns & ns & ns & $\mathrm{ns}$ & ns & - & ns & ns & ns & ns \\
\hline El Palm-Mar & ns & ns & ns & ns & ns & ns & ns & ns & - & ns & ns & ns \\
\hline Fina El Apio & ns & ns & ns & ns & ns & ns & ns & ns & ns & - & ns & ns \\
\hline Los Gigantes & ns & ns & ns & ns & ns & ns & ns & ns & ns & ns & - & ns \\
\hline El Porís & ns & ns & ns & ns & ns & ns & ns & ns & ns & ns & ns & - \\
\hline
\end{tabular}




\begin{tabular}{|c|c|c|c|c|c|c|c|}
\hline \multicolumn{8}{|c|}{$\begin{array}{c}\text { TABLA 6. RESULTADOS DEL TEST A POSTERIORI (SNK.TEST) DE LA COMP } \\
\text { POR PARES ENTRE TAMICES DENTRO DE CADA PLAYA PARA } \\
\text { LA VARIABLE NÚMERO DE MICROPLÁSTICOS }\end{array}$} \\
\hline \multicolumn{8}{|c|}{ NÚMERo } \\
\hline \multicolumn{8}{|c|}{ SNK.TEST: ENTRE TAMICES DENTRO DE CADA PLAYA } \\
\hline Las Galletas & $\begin{array}{l}\text { Tamiz } \\
4 \mathrm{~mm}\end{array}$ & $\begin{array}{l}\text { Tamiz } \\
1 \mathrm{~mm}\end{array}$ & $\begin{array}{c}\text { Tamiz } \\
0.5 \mathrm{~mm}\end{array}$ & Playa Jardín & $\begin{array}{l}\text { Tamiz } \\
4 \mathrm{~mm}\end{array}$ & $\begin{array}{l}\text { Tamiz } \\
1 \mathrm{~mm}\end{array}$ & $\begin{array}{c}\text { Tamiz } \\
0.5 \mathrm{~mm}\end{array}$ \\
\hline Tamiz $4 \mathrm{~mm}$ & - & ns & ns & Tamiz $4 \mathrm{~mm}$ & - & ns & ns \\
\hline Tamiz $1 \mathrm{~mm}$ & ns & - & ns & Tamiz $1 \mathrm{~mm}$ & ns & - & ns \\
\hline Tamiz $0.5 \mathrm{~mm}$ & ns & ns & - & Tamiz $0.5 \mathrm{~mm}$ & ns & ns & - \\
\hline La Tejita & $\begin{array}{l}\text { Tamiz } \\
4 \mathrm{~mm}\end{array}$ & $\begin{array}{l}\text { Tamiz } \\
1 \mathrm{~mm}\end{array}$ & $\begin{array}{c}\text { Tamiz } \\
0.5 \mathrm{~mm}\end{array}$ & La Caleta & $\begin{array}{l}\text { Tamiz } \\
4 \mathrm{~mm}\end{array}$ & $\begin{array}{l}\text { Tamiz } \\
1 \mathrm{~mm}\end{array}$ & $\begin{array}{c}\text { Tamiz } \\
0.5 \mathrm{~mm}\end{array}$ \\
\hline Tamiz $4 \mathrm{~mm}$ & - & ns & ns & Tamiz $4 \mathrm{~mm}$ & - & ns & ns \\
\hline Tamiz $1 \mathrm{~mm}$ & ns & - & ns & Tamiz $1 \mathrm{~mm}$ & ns & - & ns \\
\hline Tamiz $0.5 \mathrm{~mm}$ & ns & ns & - & Tamiz $0.5 \mathrm{~mm}$ & ns & ns & - \\
\hline $\begin{array}{l}\text { Punta del } \\
\text { Hidalgo }\end{array}$ & $\begin{array}{l}\text { Tamiz } \\
4 \mathrm{~mm}\end{array}$ & $\begin{array}{l}\text { Tamiz } \\
1 \mathrm{~mm}\end{array}$ & $\begin{array}{c}\text { Tamiz } \\
0.5 \mathrm{~mm}\end{array}$ & El Médano & $\begin{array}{l}\text { Tamiz } \\
4 \mathrm{~mm}\end{array}$ & $\begin{array}{l}\text { Tamiz } \\
1 \mathrm{~mm}\end{array}$ & $\begin{array}{l}\text { Tamiz } \\
0.5 \mathrm{~m}\end{array}$ \\
\hline Tamiz 4 mm & - & ns & ns & Tamiz $4 \mathrm{~mm}$ & - & ns & ns \\
\hline Tamiz $1 \mathrm{~mm}$ & ns & - & ns & Tamiz $1 \mathrm{~mm}$ & ns & - & ns \\
\hline Tamiz $0.5 \mathrm{~mm}$ & ns & ns & - & Tamiz $0.5 \mathrm{~mm}$ & ns & ns & - \\
\hline $\begin{array}{l}\text { Playa San } \\
\text { Marcos }\end{array}$ & $\begin{array}{l}\text { Tamiz } \\
4 \mathrm{~mm}\end{array}$ & $\begin{array}{l}\text { Tamiz } \\
1 \mathrm{~mm}\end{array}$ & $\begin{array}{c}\text { Tamiz } \\
0.5 \mathrm{~mm}\end{array}$ & Las Gaviotas & $\begin{array}{l}\text { Tamiz } \\
4 \mathrm{~mm}\end{array}$ & $\begin{array}{l}\text { Tamiz } \\
1 \mathrm{~mm}\end{array}$ & $\begin{array}{c}\text { Tamiz } \\
0.5 \mathrm{~mm}\end{array}$ \\
\hline Tamiz 4 mm & - & ns & ns & Tamiz $4 \mathrm{~mm}$ & - & ns & $\mathrm{ns}$ \\
\hline Tamiz $1 \mathrm{~mm}$ & ns & - & ns & Tamiz $1 \mathrm{~mm}$ & ns & - & ns \\
\hline Tamiz $0.5 \mathrm{~mm}$ & ns & ns & - & Tamiz $0.5 \mathrm{~mm}$ & ns & ns & - \\
\hline El Palm-Mar & $\begin{array}{l}\text { Tamiz } \\
4 \mathrm{~mm}\end{array}$ & $\begin{array}{l}\text { Tamiz } \\
1 \mathrm{~mm}\end{array}$ & $\begin{array}{c}\text { Tamiz } \\
0.5 \mathrm{~mm}\end{array}$ & Finca El Apio & $\begin{array}{l}\text { Tamiz } \\
4 \mathrm{~mm}\end{array}$ & $\begin{array}{l}\text { Tamiz } \\
1 \mathrm{~mm}\end{array}$ & $\begin{array}{c}\text { Tamiz } \\
0.5 \mathrm{~mm}\end{array}$ \\
\hline Tamiz 4 mm & - & ns & ns & Tamiz $4 \mathrm{~mm}$ & - & ns & ns \\
\hline Tamiz $1 \mathrm{~mm}$ & ns & - & ns & Tamiz $1 \mathrm{~mm}$ & ns & - & ns \\
\hline Tamiz $0.5 \mathrm{~mm}$ & ns & ns & - & Tamiz $0.5 \mathrm{~mm}$ & ns & ns & - \\
\hline Los Gigantes & $\begin{array}{l}\text { Tamiz } \\
4 \mathrm{~mm}\end{array}$ & $\begin{array}{l}\text { Tamiz } \\
1 \mathrm{~mm}\end{array}$ & $\begin{array}{c}\text { Tamiz } \\
0.5 \mathrm{~mm}\end{array}$ & El Porís & $\begin{array}{l}\text { Tamiz } \\
4 \mathrm{~mm}\end{array}$ & $\begin{array}{l}\text { Tamiz } \\
1 \mathrm{~mm}\end{array}$ & $\begin{array}{c}\text { Tamiz } \\
0.5 \mathrm{~mm}\end{array}$ \\
\hline Tamiz 4 mm & - & $* * *$ & ns & Tamiz 4 mm & - & ns & ns \\
\hline Tamiz $1 \mathrm{~mm}$ & $* * *$ & - & $* * *$ & Tamiz $1 \mathrm{~mm}$ & ns & - & ns \\
\hline Tamiz $0.5 \mathrm{~mm}$ & ns & $* * *$ & - & Tamiz $0.5 \mathrm{~mm}$ & ns & ns & - \\
\hline
\end{tabular}

Los niveles de significación fueron: ns $\mathrm{p}>0.05,{ }^{*} \mathrm{p}<0.05,{ }^{* *} \mathrm{p}<0.01 \mathrm{y}{ }^{* * *} \mathrm{p}<0.001$ ). 


\begin{tabular}{|c|c|c|c|}
\hline \multicolumn{4}{|c|}{$\begin{array}{c}\text { TABLA 7. RESULTADOS DEL TEST A POSTERIORI (SNK.TEST) DE LA COMPARACIÓN } \\
\text { POR PARES ENTRE TRANSECTOS DENTRO DE CADA TAMIZ PARA } \\
\text { LA VARIABLE NÚMERO DE MICROPLÁSTICOS }\end{array}$} \\
\hline \multicolumn{4}{|c|}{ NúMERo } \\
\hline \multicolumn{4}{|c|}{ SNK.TEST: ENTRE TRANSECTOS DENTRO DE CADA TAMIZ } \\
\hline Tamiz $4 \mathrm{~mm}$ & Transecto 1 & Transecto 2 & Transecto 3 \\
\hline Transecto 1 & - & ns & ns \\
\hline Transecto 2 & ns & - & ns \\
\hline Transecto 3 & ns & ns & - \\
\hline Tamiz $1 \mathrm{~mm}$ & Transecto 1 & Transecto 2 & Transecto 3 \\
\hline Transecto 1 & - & ns & ns \\
\hline Transecto 2 & $\mathrm{~ns}$ & - & ns \\
\hline Transecto 3 & ns & ns & - \\
\hline Tamiz $0.5 \mathrm{~mm}$ & Transecto 1 & Transecto 2 & Transecto 3 \\
\hline Transecto 1 & - & ns & ns \\
\hline Transecto 2 & ns & - & ns \\
\hline Transecto 3 & ns & $\mathrm{ns}$ & - \\
\hline
\end{tabular}

Los niveles de significación fueron: $\left.\mathrm{ns} \mathrm{p}>0.05,{ }^{*} \mathrm{p}<0.05,{ }^{* *} \mathrm{p}<0.01 \mathrm{y}{ }^{* * *} \mathrm{p}<0.001\right)$.

\begin{tabular}{|c|c|c|c|}
\hline \multicolumn{4}{|c|}{$\begin{array}{l}\text { TABLA 8. RESULTADOS DEL TEST A POSTERIORI (SNK.TEST) DE LA } \\
\text { POR PARES ENTRE TAMICES DENTRO DE CADA TRANSEC } \\
\text { LA VARIABLE NÚMERO DE MICROPLÁSTICOS }\end{array}$} \\
\hline \multicolumn{4}{|c|}{ NÚMERO } \\
\hline \multicolumn{4}{|c|}{ SNK.TEST: ENTRE TAMICES DENTRO DE CADA TRANSECTO } \\
\hline Transecto 1 & Tamiz $4 \mathrm{~mm}$ & Tamiz $1 \mathrm{~mm}$ & Tamiz $0.5 \mathrm{~mm}$ \\
\hline Tamiz $4 \mathrm{~mm}$ & - & ns & ns \\
\hline Tamiz $1 \mathrm{~mm}$ & ns & - & ns \\
\hline Tamiz $0.5 \mathrm{~mm}$ & ns & ns & - \\
\hline Transecto 2 & Tamiz $4 \mathrm{~mm}$ & Tamiz $1 \mathrm{~mm}$ & Tamiz $0.5 \mathrm{~mm}$ \\
\hline Tamiz $4 \mathrm{~mm}$ & - & ns & ns \\
\hline Tamiz $1 \mathrm{~mm}$ & ns & - & ns \\
\hline Tamiz $0.5 \mathrm{~mm}$ & ns & ns & - \\
\hline Transecto 3 & Tamiz $4 \mathrm{~mm}$ & Tamiz $1 \mathrm{~mm}$ & Tamiz $0.5 \mathrm{~mm}$ \\
\hline Tamiz $4 \mathrm{~mm}$ & - & ns & ns \\
\hline Tamiz $1 \mathrm{~mm}$ & ns & - & ns \\
\hline Tamiz $0.5 \mathrm{~mm}$ & ns & ns & - \\
\hline
\end{tabular}

Los niveles de significación fueron: ns $\left.\mathrm{p}>0.05,{ }^{*} \mathrm{p}<0.05,{ }^{* *} \mathrm{p}<0.01 \mathrm{y}{ }^{* * *} \mathrm{p}<0.001\right)$. 

POR PARES ENTRE PLAYAS DENTRO DE CADA TRANSECTO PARA

LA VARIABLE PESO DE MICROPLÁSTICOS

Peso

SNK.TEST: ENTRE PLAYAS DENTRO DE CADA TRANSECTO

Transecto 1 㺃

Las Galletas - ns ns ns ns ns ns ns ns ns ns ns

$\begin{array}{llllllllllllllll}\text { Playa Jardín } & \mathrm{ns} & - & \mathrm{ns} & \mathrm{ns} & \mathrm{ns} & \mathrm{ns} & \mathrm{ns} & \mathrm{ns} & \mathrm{ns} & \mathrm{ns} & \mathrm{ns} & \mathrm{ns}\end{array}$

$\begin{array}{lllllllllllllll}\text { La Tejita } & \mathrm{ns} & \mathrm{ns} & - & \mathrm{ns} & \mathrm{ns} & \mathrm{ns} & \mathrm{ns} & \mathrm{ns} & \mathrm{ns} & \mathrm{ns} & \mathrm{ns} & \mathrm{ns}\end{array}$

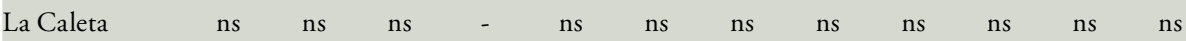

Punta del

Hidalgo

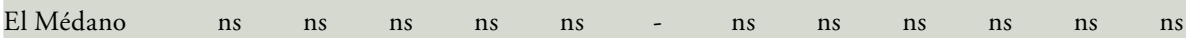

Playa San $n s$ ns ns ns ns ns - ns ns ns ns ns

Marcos

$\mathrm{ns} \quad \mathrm{ns} \quad \mathrm{ns} \quad \mathrm{ns} \quad \mathrm{ns} \quad$ ns

$\begin{array}{llllllllllllllllllllll}\text { Las Gaviotas } & \mathrm{ns} & \mathrm{ns} & \mathrm{ns} & \mathrm{ns} & \mathrm{ns} & \mathrm{ns} & \mathrm{ns} & - & \mathrm{ns} & \mathrm{ns} & \mathrm{ns} & \mathrm{ns}\end{array}$

El Palm-Mar ns ns ns ns ns ns $n s \quad$ ns $\quad$ ns $\quad$ ns $\quad$ ns ns

Finca El Apio $n s \quad n s \quad n s \quad n s \quad n s \quad n s \quad n s \quad n s \quad n s \quad-\quad n s \quad n s$

Los Gigantes ns ns ns ns ns ns $n s \quad$ ns $n s \quad$ ns $\quad-\quad n s$

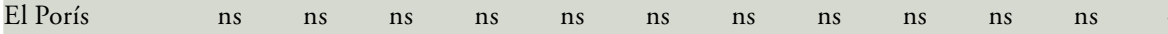

Transecto 2 总

Las Galletas - ns ns ns ns ns ns ns ns ns ns ns

Playa Jardín ns $\quad$ ns ns ns ns $n s \quad$ ns $n s \quad$ ns $n s \quad$ ns

$\begin{array}{llllllllllllll}\text { La Tejita } & \text { ns } & \text { ns } & - & & \text { ns } & \text { ns } & \text { ns } & \text { ns } & \text { ns } & \text { ns } & * & & \text { ns }\end{array}$

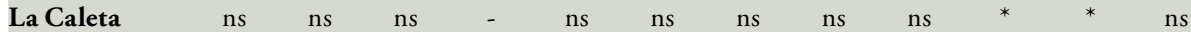

Punta del ns ns ns ns - $\quad$ ns ns ns ns ns ns ns

Hidalgo

$\begin{array}{llllllllllllllll}\text { El Médano } & n s & n s & n s & n s & n s & - & n s & n s & n s & n s\end{array}$

Playa San $n s \quad n s \quad n s \quad n s \quad n s \quad n s \quad-\quad n s \quad n s \quad * \quad * \quad$ ns

Marcos

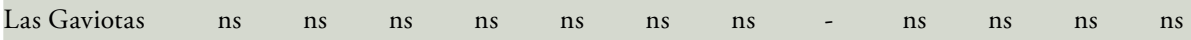

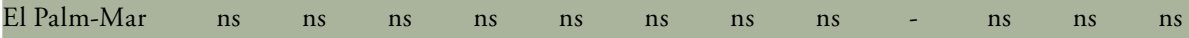

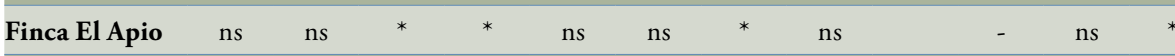

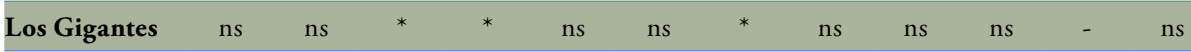

$\begin{array}{llllllllllllll}\text { El Porís } & \mathrm{ns} & \mathrm{ns} & \mathrm{ns} & \mathrm{ns} & \mathrm{ns} & \mathrm{ns} & \mathrm{ns} & \mathrm{ns} & \mathrm{ns} & * & \mathrm{~ns} & -\end{array}$




\begin{tabular}{|c|c|c|c|c|c|c|c|c|c|c|c|c|}
\hline Transecto 3 & $\underset{\Xi}{\stackrel{\Xi}{\Xi}}$ & 奈恶 & 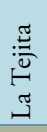 & $\underset{\widetilde{J}}{\frac{\pi}{\sim}}$ & 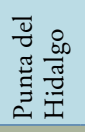 & 푀 & 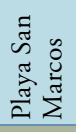 & $\stackrel{\overbrace{}}{\stackrel{\pi}{\leftrightarrows}}$ & 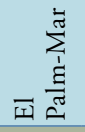 & 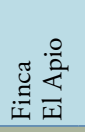 & 总 & 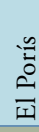 \\
\hline Las Galletas & - & ns & $\mathrm{ns}$ & ns & ns & $\mathrm{ns}$ & ns & ns & ns & ns & ns & ns \\
\hline Playa Jardín & ns & - & $\mathrm{ns}$ & ns & ns & ns & ns & ns & ns & ns & ns & ns \\
\hline La Tejita & ns & ns & - & ns & ns & ns & ns & ns & ns & ns & ns & ns \\
\hline La Caleta & ns & ns & ns & - & ns & ns & ns & ns & ns & ns & ns & ns \\
\hline $\begin{array}{l}\text { Punta del } \\
\text { Hidalgo }\end{array}$ & ns & ns & ns & ns & - & ns & ns & ns & ns & ns & $\mathrm{ns}$ & ns \\
\hline El Médano & ns & ns & ns & ns & ns & - & ns & ns & ns & ns & ns & ns \\
\hline $\begin{array}{l}\text { Playa San } \\
\text { Marcos }\end{array}$ & ns & ns & ns & ns & ns & $\mathrm{ns}$ & - & ns & ns & $\mathrm{ns}$ & ns & ns \\
\hline Las Gaviotas & ns & ns & ns & ns & ns & ns & ns & - & ns & ns & ns & ns \\
\hline El Palm-Mar & ns & ns & ns & $\mathrm{ns}$ & ns & ns & ns & ns & - & ns & ns & ns \\
\hline Finca El Apio & ns & ns & ns & ns & ns & ns & ns & ns & ns & - & ns & ns \\
\hline Los Gigantes & ns & ns & ns & $\mathrm{ns}$ & ns & ns & $\mathrm{ns}$ & ns & ns & ns & - & $\mathrm{ns}$ \\
\hline El Porís & ns & ns & ns & ns & ns & ns & $\mathrm{ns}$ & ns & ns & ns & ns & - \\
\hline
\end{tabular}

Los niveles de significación fueron: ns $\left.\mathrm{p}>0.05,{ }^{*} \mathrm{p}<0.05,{ }^{* *} \mathrm{p}<0.01 \mathrm{y}{ }^{* * *} \mathrm{p}<0.001\right)$.

\begin{tabular}{|c|c|c|c|c|c|c|c|}
\hline \multicolumn{8}{|c|}{$\begin{array}{c}\text { TABLA 10. RESULTADOS DEL TEST A POSTERIORI (SNK.TEST) DE LA COMPARACIÓN } \\
\text { POR PARES ENTRE TRANSECTOS DENTRO DE CADA PLAYA PARA } \\
\text { LA VARIABLE PESO DE MICROPLÁSTICOS }\end{array}$} \\
\hline \multicolumn{8}{|c|}{ Peso } \\
\hline \multicolumn{8}{|c|}{ SNK.TEST: ENTRE TRANSECTOS DENTRO DE CADA PLAYA } \\
\hline Las Galletas & 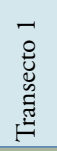 & 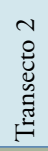 & 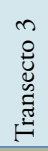 & Playa Jardín & 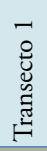 & 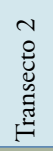 & 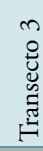 \\
\hline Transecto 1 & - & ns & ns & Transecto 1 & - & ns & ns \\
\hline Transecto 2 & ns & - & ns & Transecto 2 & ns & - & ns \\
\hline Transecto 3 & ns & ns & - & Transecto 3 & ns & ns & - \\
\hline La Tejita & 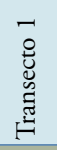 & 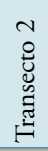 & 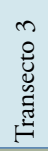 & La Caleta & 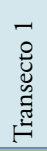 & 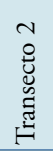 & 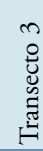 \\
\hline Transecto 1 & - & ns & ns & Transecto 1 & - & ns & ns \\
\hline Transecto 2 & ns & - & ns & Transecto 2 & ns & - & $\mathrm{ns}$ \\
\hline Transecto 3 & ns & ns & - & Transecto 3 & ns & ns & - \\
\hline
\end{tabular}




\begin{tabular}{|c|c|c|c|c|c|c|c|}
\hline $\begin{array}{l}\text { Punta del } \\
\text { Hidalgo }\end{array}$ & 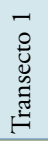 & 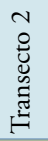 & 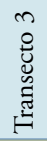 & El Médano & 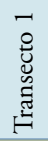 & 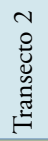 & 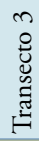 \\
\hline Transecto 1 & - & ns & ns & Transecto 1 & - & ns & ns \\
\hline Transecto 2 & ns & - & $\mathrm{ns}$ & Transecto 2 & ns & - & ns \\
\hline Transecto 3 & ns & ns & - & Transecto 3 & ns & ns & - \\
\hline $\begin{array}{l}\text { Playa San } \\
\text { Marcos }\end{array}$ & 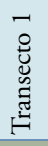 & 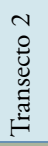 & 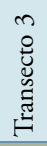 & Las Gaviotas & 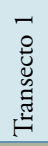 & 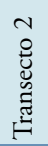 & 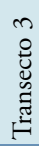 \\
\hline Transecto 1 & - & ns & ns & Transecto 1 & - & ns & ns \\
\hline Transecto 2 & ns & - & ns & Transecto 2 & ns & - & ns \\
\hline Transecto 3 & ns & ns & - & Transecto 3 & ns & ns & - \\
\hline El Palm-Mar & 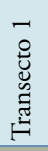 & 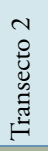 & 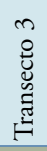 & Finca El Apio & 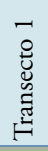 & 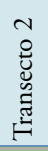 & 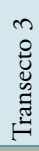 \\
\hline Transecto 1 & - & ns & ns & Transecto 1 & - & ns & ns \\
\hline Transecto 2 & ns & - & ns & Transecto 2 & ns & - & ns \\
\hline Transecto 3 & ns & ns & - & Transecto 3 & ns & ns & - \\
\hline Los Gigantes & 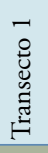 & 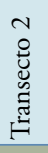 & 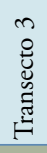 & El Porís & 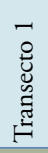 & 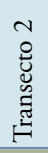 & 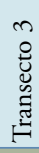 \\
\hline Transecto 1 & - & ns & * & Transecto 1 & - & ns & ns \\
\hline Transecto 2 & ns & - & ns & Transecto 2 & ns & - & ns \\
\hline Transecto 3 & $*$ & ns & - & Transecto 3 & ns & ns & - \\
\hline
\end{tabular}

Los niveles de significación fueron: $\left.\mathrm{ns} \mathrm{p}>0.05,{ }^{*} \mathrm{p}<0.05,{ }^{* *} \mathrm{p}<0.01 \mathrm{y}{ }^{* * *} \mathrm{p}<0.001\right)$. 
